\title{
Thermodynamically stable [4 + 2] cycloadducts of lanthanum-encapsulated endohedral metallofullerenes
}

\author{
Yuta Takano ${ }^{1}$, Yuki Nagashima ${ }^{2}$, M. Ángeles Herranz ${ }^{3}$, Nazario Martín ${ }^{* 3,4}$ \\ and Takeshi Akasaka*2,5,6
}

\section{Full Research Paper}

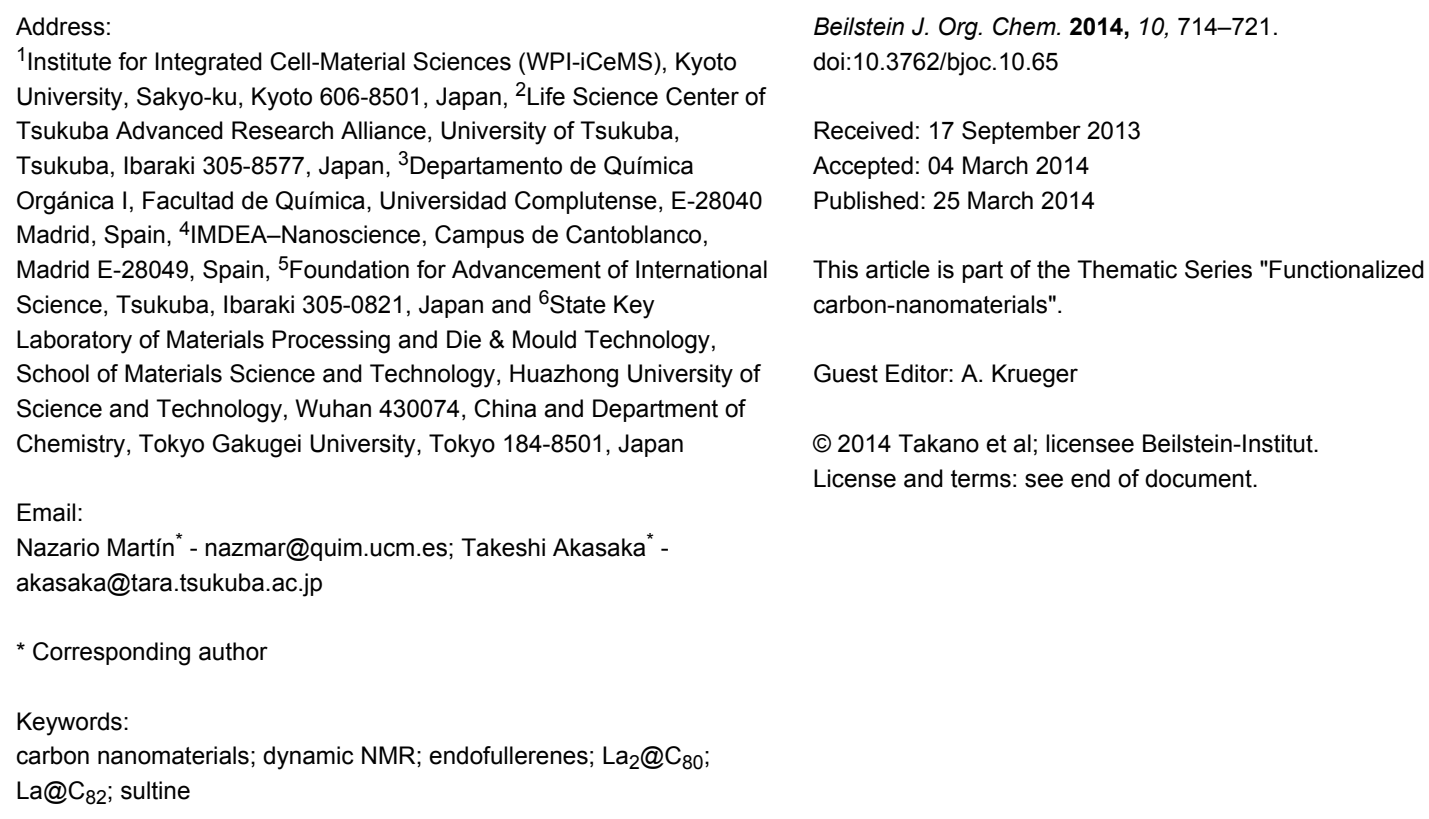




\section{Introduction}

Endohedral metallofullerenes (EMFs) are a family of nanocarbons, which encapsulated one or more metal atoms inside a hollow carbon cage [1-4]. The encapsulation results in the electron transfer from metal atoms to the fullerene cage, which leads to unique electronic, magnetic, and chemical properties for EMFs that cannot be expected for empty fullerenes. Due to the numerous electronic properties EMFs are anticipated as promising materials in various fields such as chemistry, biology, and material science.

Among various kinds of EMFs, those encapsulating La atoms are especially attractive molecules because of their electronic and magnetic characteristics. As a result of the three electron transfer per La atom to the fullerene cage, the fullerenes simultaneously possess a low ionizing potential and a high electron affinity [1,2]. For mono-La endohedral fullerenes such as $\mathrm{La} @ \mathrm{C}_{82}$, the electron transfer results in paramagnetism of the fullerene cage [5]. The di-La endohedral fullerenes such as $\mathrm{La}_{2} @ \mathrm{C}_{80}$ show diamagnetism [6]. This feature leads to remarkable differences in chemical and electronic properties between these two classes of EMFs.

Chemical functionalization of fullerenes enhances molecular properties and possible applications of fullerenes $[1,4]$. The $[4+2]$ cycloaddition reaction is a useful chemical modification method because it enables to introduce a variety of addends and/or the combination of different functionalities on the fullerene [7]. Regarding the [4+2] cycloaddition of EMFs, however, no report is available for endohedral di-metallofullerenes, with the exception of azafullerene [8]. A limited number of reports describe other EMFs $[4,9,10]$. Moreover, the only precedent of $[4+2]$ cycloadducts of the fullerenes which have an open-shell electronic structure of the cage, e.g., La@ $\mathrm{C}_{82}$, are thermodynamically unstable and show retrocycloaddition reactions $[10,11]$. In addition, boat-to-boat inver- sion of the addend of the cycloadducts of EMFs has not been well-studied to date; the investigation of this interconversion serves to demonstrate the existence of a dynamic process in the molecules and is regarded as one index of the bonding energy of the addition position of the fullerene and the addend.

Among various precursors to afford [4 +2$]$ cycloadducts of fullerenes, the sultine 4,5-benzo-3,6-dihydro-1,2-oxathiin 2-oxide and its derivatives are useful to afford thermodynamically stable compounds because thermolysis of sultine affords highly reactive $o$-quinodimethanes by extrusion of sulfur dioxide without production of any organic or inorganic byproduct $[12,13]$.

Here, we present the first chemical derivatization of $\mathrm{La}_{2} @ \mathrm{C}_{80}$ and La@ $\mathrm{C}_{82}$ by [4+2] cycloaddition using sultines 1a,b. The resulting products $\mathbf{3 a}, \mathbf{b}, \mathbf{4} \mathbf{a}, \mathbf{b}$ and $\mathbf{5 b}$ were characterized and their thermodynamic properties were investigated.

\section{Results and Discussion Synthesis and characterization of $\mathrm{La}_{2} @ \mathrm{C}_{80}$ cycloadducts}

$o$-Quinodimethanes $\mathbf{2 a}$ and $\mathbf{2} \mathbf{b}$ were generated in situ by thermolysis of the corresponding sultines $\mathbf{1 a}$ and $\mathbf{1 b}$ in toluene at $80{ }^{\circ} \mathrm{C}$ (Scheme 1). The highly reactive intermediates are trapped efficiently by $\mathrm{La}_{2} @ \mathrm{C}_{80}$, which acts as a dienophile to form the cycloadducts (3a,b and $\mathbf{4 a}, \mathbf{b})$. The reactions were traced using HPLC analyses (Figure 1), and formation of the resulting $[4+2]$ adducts was confirmed by matrix-assisted laser desorption ionization (MALDI) TOF mass spectrometry (Figure 2), which shows the molecular ion peaks for the corresponding compounds. Isolation of $\mathbf{3 b}$ and $\mathbf{4 b}$ were achieved by one-step HPLC separation using a Buckyprep column, although this purification method was not applicable for 3a and $\mathbf{4 a}$ (vide infra).<smiles>[R]c1ccc([R])c2c1COS(=O)C2</smiles>
toluene, $80^{\circ} \mathrm{C}$

$1 a, b$

a: $\mathrm{R}=\mathrm{H}$

b: $\mathrm{R}=\mathrm{OMe}$
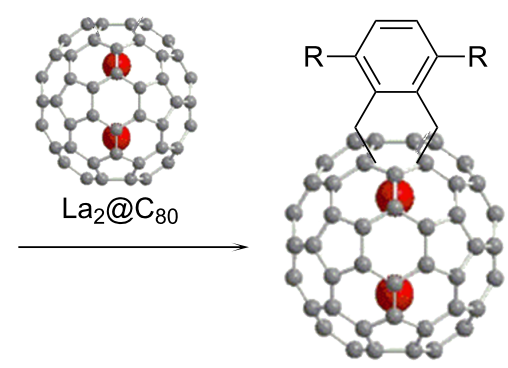

3a,b: $[6,6]$-adducts 4a,b: $[5,6]$-adducts

Scheme 1: Synthesis of $[4+2]$ adducts of $L_{2} @ C_{80}$. 
(a)

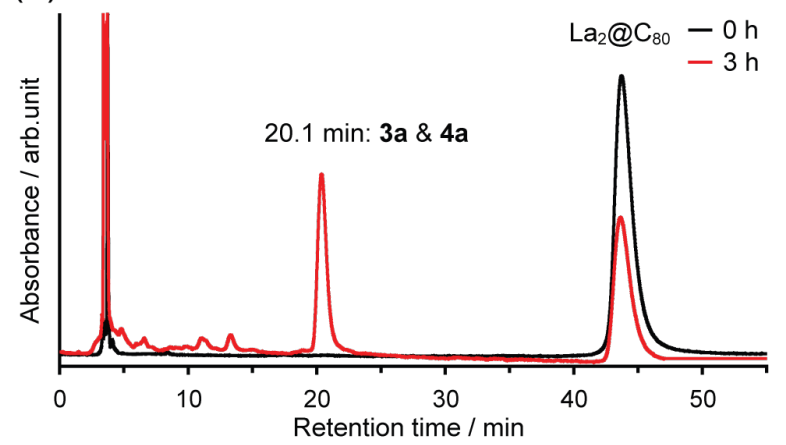

(b)

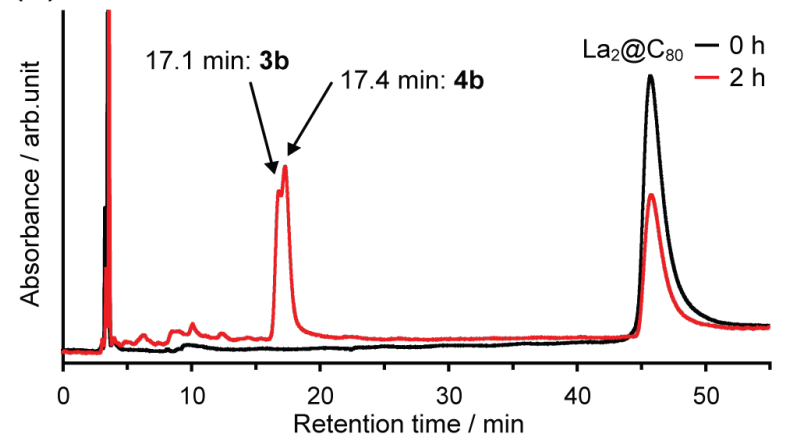

Figure 1: HPLC profiles of the reaction solutions (black) before and (red) after the reaction of $\mathrm{La}_{2} @ \mathrm{C}_{80}$ and (a) $1 \mathrm{a}$ and (b) 1b, respectively. Conditions: column, Buckyprep ( $\varnothing 4.6 \mathrm{~mm} \times 250 \mathrm{~mm}$ ); eluent, toluene; flow rate, $1.0 \mathrm{~mL} / \mathrm{min}$; wavelength: $330 \mathrm{~nm}$; rt.

Because only two types of $\mathrm{C}=\mathrm{C}$ bonds are available in $\mathrm{La}_{2} @ \mathrm{C}_{80}$, which has $I_{h}$ symmetry, only two site-isomers namely $[6,6]$ - and [5,6]-isomers, are allowed to be formed by cycloaddition reactions. (Please note that "site-isomer" refers to an isomer of the adducts which has the same fullerene and addend but different addition position - a classification proposed recently for fullerene's chemistry by Martin et al. [14]). Therefore, $\mathbf{3 b}$ and $\mathbf{4 b}$ are concluded to be the site-isomers which were afforded by the reaction as a result of using highly reactive $o$-quinodimethane.

The UV-vis spectra of $\mathbf{3 b}$ and $\mathbf{4 b}$ partially provide information related to their molecular structures. The spectra were firstly recorded using a diode-array detector of the HPLC apparatus (Figure 3). The spectrum of $\mathbf{3 b}$ shows the specific absorption band around $700 \mathrm{~nm}$, which strongly suggests the electric nature of a [6,6]-closed adduct of the $\mathrm{La}_{2} @ \mathrm{C}_{80}$ derivatives [15] because the absorption spectra of fullerenes and their derivatives are mainly attributable to $\pi-\pi^{*}$ transitions, which reflects the distinctive fingerprints of the $\pi$-electron system topology of the fullerene cage. Similarly, the characteristic spectrum of $\mathbf{4 b}$ suggests its [5,6]-closed structure.

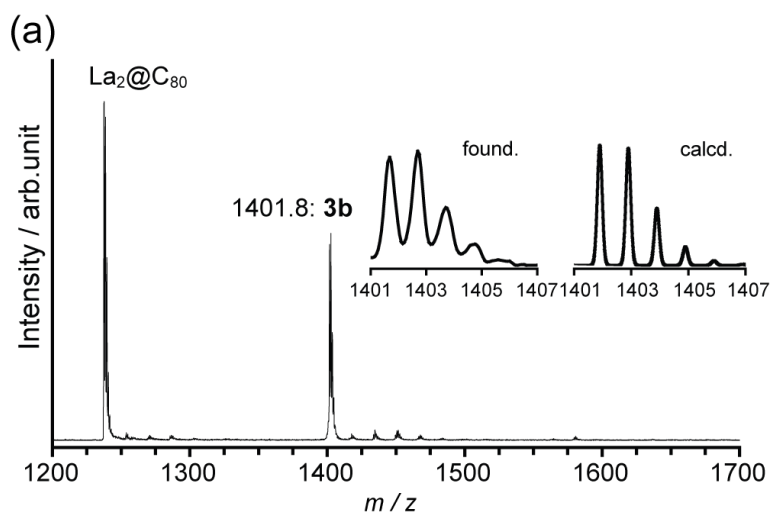

(b)

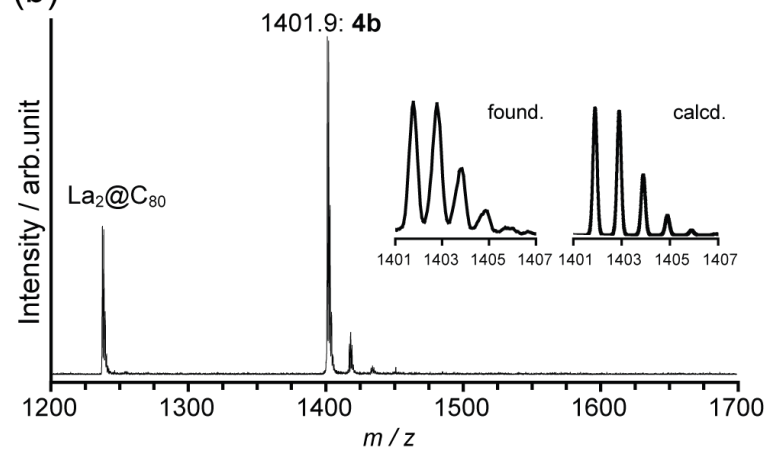

Figure 2: MALDI-TOF mass (negative mode) spectra of (a) $\mathbf{3 b}$ and (b) 4b, using 1,1,4,4-tetraphenyl-1,3-butadiene as matrix.

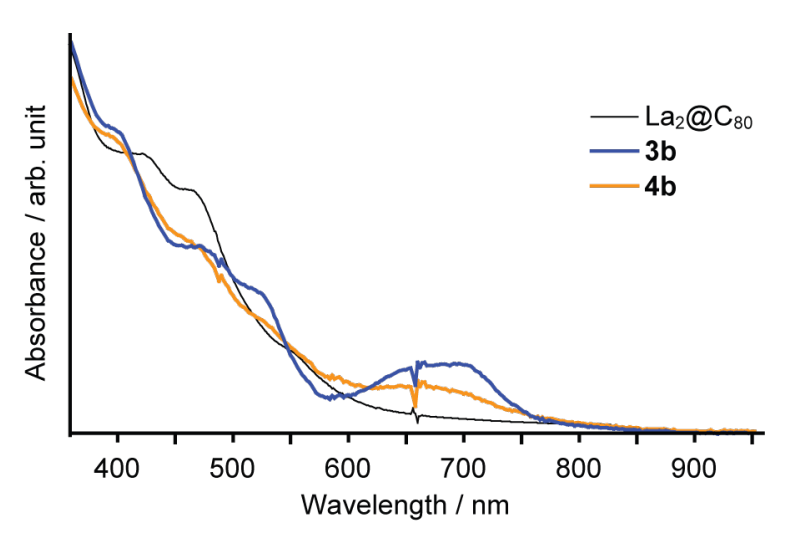

Figure 3: UV-vis/near-IR absorption spectra of $\mathbf{3 b}$ and $\mathbf{4 b}$ recorded by the diode array detector of the HPLC apparatus.

A different approach was taken to purify $\mathbf{3 a}$ and $\mathbf{4 a}$ because of the similar retention time of these compounds in HPLC. The mixture of 3a and 4a was first separated from the unreacted starting materials and byproducts through one-step HPLC separation. The MALDI-TOF mass spectra of $\mathbf{3 a}$ and $4 \mathbf{a}$ showed single peaks attributed to the molecular ion peak of the target molecule, $\mathrm{La}_{2} @ \mathrm{C}_{80} \mathrm{C}_{2} \mathrm{H}_{4} \mathrm{C}_{6} \mathrm{H}_{4}$, at $1342 \mathrm{~m} / z$ (Figure 4). The existence of both $[6,6]$ - and $[5,6]$-isomers in the mixture was 
indicated by the ${ }^{1} \mathrm{H}$ NMR spectrum recorded at $248 \mathrm{~K}$ (Figure $5 \mathrm{~b}$ ), which cannot be expected from a single regioisomer. The $[6,6]$-adduct should show only two sets of $A B$ quartets. The $[5,6]$-adduct should demonstrate one or two $\mathrm{AB}$ quartets based on its $C_{S}$ molecular symmetry. Consequently, the spectrum containing more than two $\mathrm{AB}$ quartets indicates the existence of the both site-isomers.

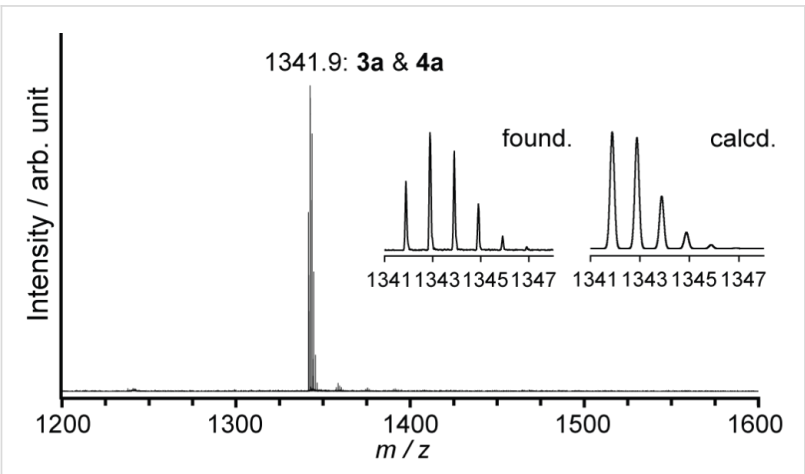

Figure 4: MALDI-TOF mass spectrum (negative mode) of the reaction mixture from $\mathrm{La}_{2} @ \mathrm{C}_{80}$ and 1a, using 1,1,4,4-tetraphenyl-1,3-butadiene as matrix.

(a)

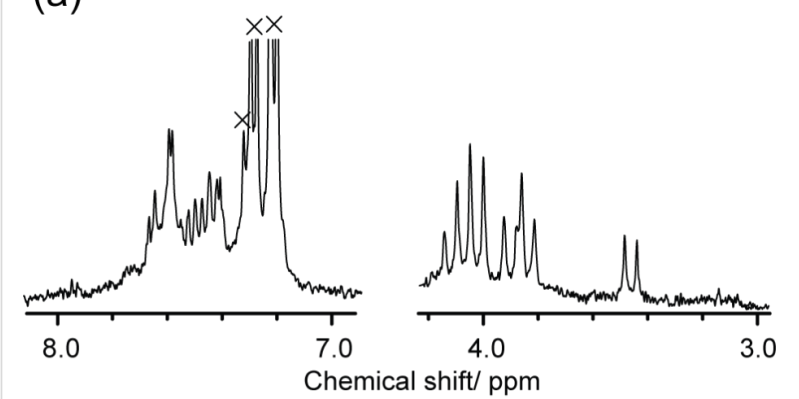

(b)

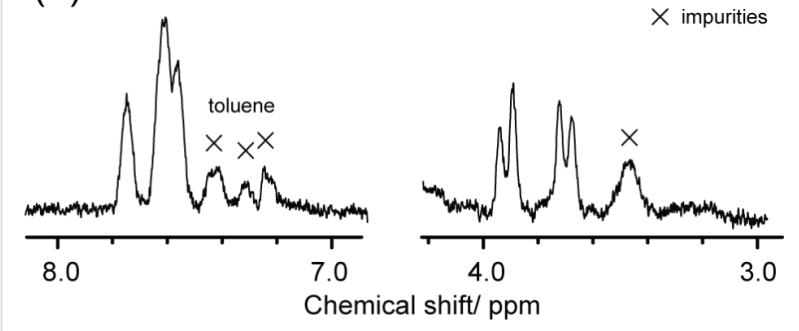

Figure 5: ${ }^{1} \mathrm{H}$ NMR spectra of (a) the mixture of $3 a$ and $4 a$ in $\mathrm{C}_{2} \mathrm{D}_{2} \mathrm{Cl}_{4}$ at $248 \mathrm{~K}$, and (b) isolated $4 \mathrm{a}$ at $230 \mathrm{~K}$, recorded in $300 \mathrm{MHz}$.

Further isolation of $\mathbf{4 a}$ and $\mathbf{3 b}$ was respectively accomplished using a combination of heating and HPLC separation. When the powdery mixture of $\mathbf{3 a}$ and $\mathbf{4 a}$ was heated to a temperature of $250{ }^{\circ} \mathrm{C}$, selective decomposition of $\mathbf{3 a}$ was observed (Figure 6). Since the peak of pristine La@ $\mathrm{C}_{82}$ at ca. 30 min is observed after heating (see Figure 6b), most probably the detachment of the addend is taking place and a thermal isomerization thereafter. After HPLC purification of the crude reaction mixture, the number of the ${ }^{1} \mathrm{H}$ NMR signals attributed to the methylene protons was reduced (Figure 5b). Furthermore, the ${ }^{1} \mathrm{H}$ NMR spectrum of $4 \mathbf{a}$ at $230 \mathrm{~K}$ unambiguously shows the existence of a single regioisomer, a [5,6]-adduct, which has $C_{S}$ symmetry showing one $\mathrm{AB}$ quartet of the one set of equivalent methylene protons. The existence of the $[6,6]$-adduct is excluded because the adduct must show at least two $\mathrm{AB}$ quartets based on its molecular symmetry.

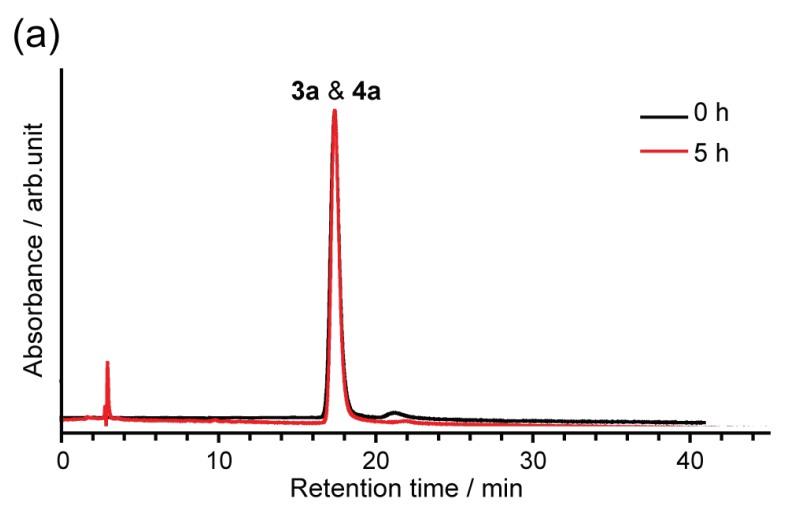

(b)

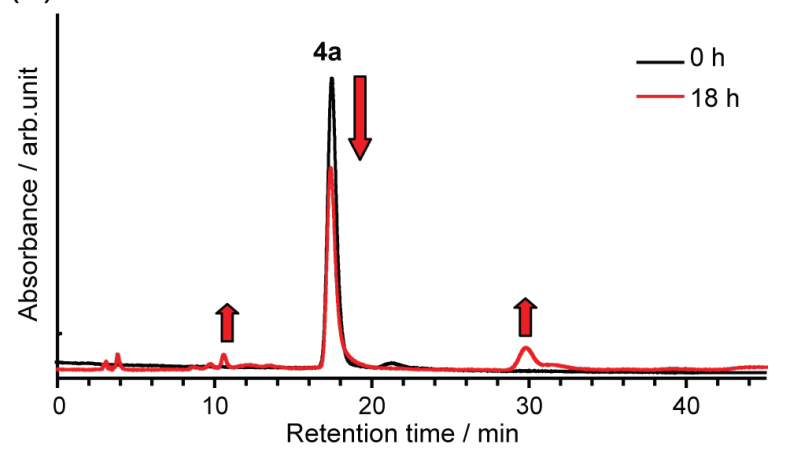

Figure 6: HPLC profiles of the mixture of $3 \mathbf{a}$ and $\mathbf{4 a}$, (a) after heating in refluxing 1,2-dichlorobenzene and (b) after heating at $250{ }^{\circ} \mathrm{C}$. Conditions: column, Buckyprep ( $\varnothing 4.6 \mathrm{~mm} \times 250 \mathrm{~mm}$ ); eluent, toluene; flow rate, $1.0 \mathrm{~mL} / \mathrm{min}$; wavelength, $330 \mathrm{~nm}$; rt.

In the case of purifying $\mathbf{3 b}$, selective decomposition of $\mathbf{4 b}$ was observed at much lower temperature than that of $\mathbf{3 a}$ and $\mathbf{4 a}$, after refluxing the mixture of $\mathbf{3 b}$ and $\mathbf{4 b}$ in toluene (Figure 7). This phenomenon is rationalized by the decomposition of the addend itself, because no pristine La@ $\mathrm{C}_{82}$ was detected after the heating in contrast to the case of $\mathbf{3 a}$ and $\mathbf{4 a}$ (vide supra). Therefore, it is concluded that the addend of $\mathbf{4} \mathbf{b}$ containing methoxy groups is thermally less stable than the addend of $\mathbf{3 a}$ and $\mathbf{4 a}$. The following HPLC purification afforded isolated $\mathbf{3 b}$. This result suggests that $\mathbf{3 b}$ is more stable against heating than $4 b$. 


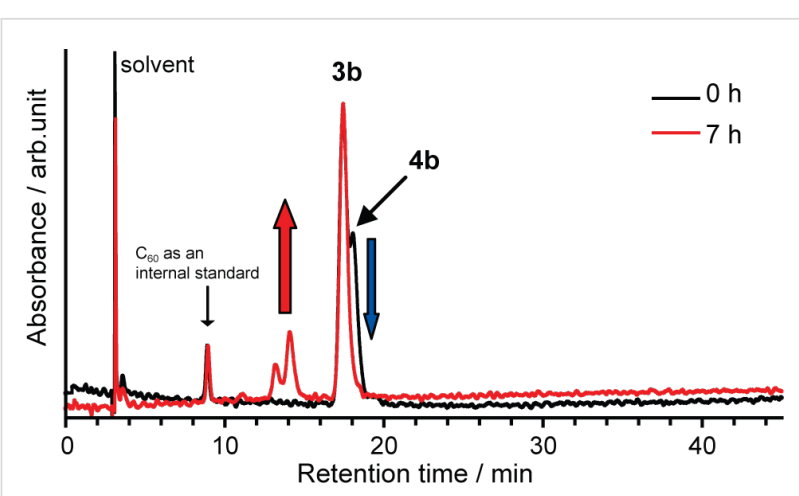

Figure 7: HPLC profiles of the reaction mixture of $\mathbf{3 b}$ and $\mathbf{4 b}$, (black) before and (red) after heating in refluxing toluene. Conditions: column, Buckyprep (Ø $4.6 \mathrm{~mm} \times 250 \mathrm{~mm}$ ); eluent, toluene; flow rate, $1.0 \mathrm{~mL} / \mathrm{min}$; wavelength, $330 \mathrm{~nm}$; rt.

The UV-vis spectra of purified $\mathbf{4 a}$ and $\mathbf{3 b}$ were recorded using a spectrophotometer (UV-3150; Shimadzu Corp.) instead of the HPLC apparatus (Figure 8). The spectra reveal that 4 a shows no clear absorption peaks in the measuring range, and demonstrates a similar spectrum to those of [5,6]-adducts of $\mathrm{La}_{2} @ \mathrm{C}_{80}$ [15]. This result shows good agreement with the ${ }^{1} \mathrm{H}$ NMR spectrum, which indicates that $\mathbf{4 a}$ is a [5,6]-adduct. However, $\mathbf{3 b}$ shows a specific absorption band around $700 \mathrm{~nm}$ as in the spectrum recorded by the HPLC system shown in Figure 3, indicating its $[6,6]$-structure.

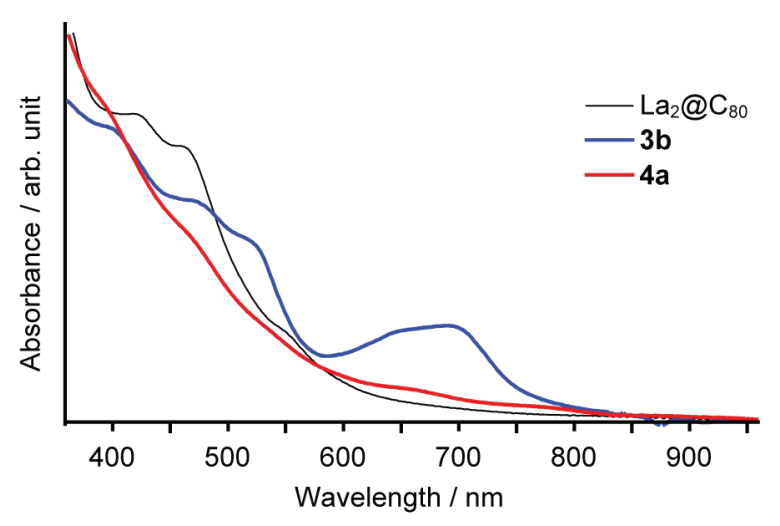

Figure 8: UV-vis/near-IR absorption spectra of $\mathbf{3 b}$ and $\mathbf{4 a}$ in toluene.

Temperature-dependent dynamics of the [4 +2$]$ adducts of endohedral metallofullerenes were studied for $\mathbf{4 a}$ by dynamic ${ }^{1} \mathrm{H}$ NMR measurements. Although 4a did not show clear peaks at $290 \mathrm{~K}$, distinct peaks were observed when the temperature was sufficiently lower or high enough distant from the coalescence temperature $\left(T_{c}\right)$ (Figure 9). This fact suggests that the boat-to-boat inversions of $\mathbf{4} \mathbf{a}$ between the pentagon side and the hexagon side are sufficiently slow or fast to allow their observation in an NMR time scale. The signals from the AB-quartet of 4a coalesce at $290 \mathrm{~K}\left(=T_{\mathrm{c}}\right)$ indicate a dynamic process, which is attributed to the boat-to-boat interconversion of the cyclohexane ring of the addend similarly to the related carbocyclic analogues of $\mathrm{C}_{60}$ adducts [12].

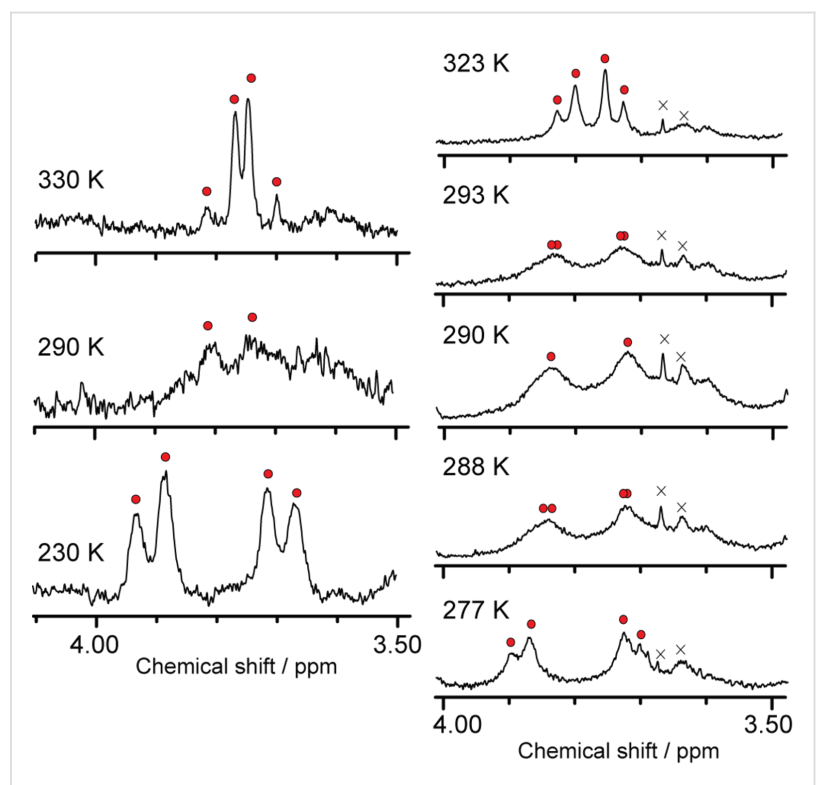

Figure 9: Temperature-dependent ${ }^{1} \mathrm{H}$ NMR spectra of $4 \mathrm{a}$ in $\mathrm{C}_{2} \mathrm{D}_{2} \mathrm{Cl}_{4}$ (left) at $300 \mathrm{MHz}$, and (right) at $500 \mathrm{MHz}$ for precise analysis.

\section{Synthesis and characterization of La@ $\mathrm{C}_{82}$ cycloadducts}

Thermal reactions of $\mathbf{1 b}$ and $\mathrm{La} @ \mathrm{C}_{82}$ afforded the [4 2] adduct 5b (Scheme 2). 5b was separated from the unreacted starting materials and byproducts through a one-step HPLC procedure (Figure 10). The MALDI-TOF mass spectrum of $\mathbf{5 b}$ shows the peak attributed to the molecular ion peak (Figure 11).

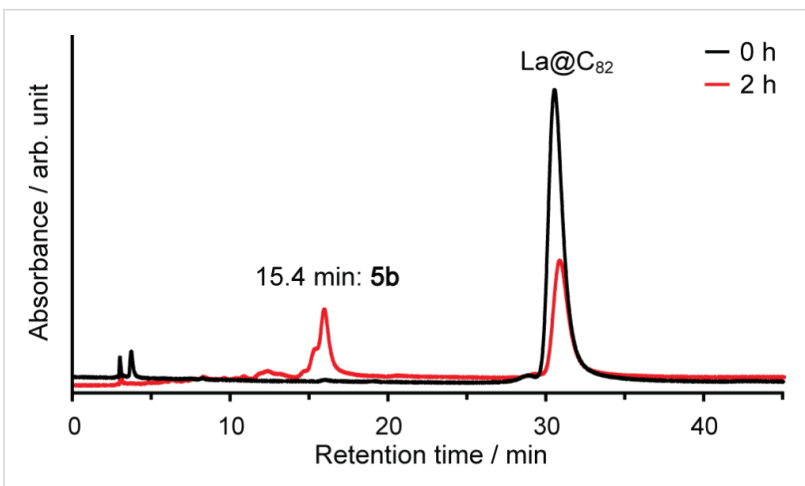

Figure 10: HPLC profiles of the reaction mixture for $\mathbf{5 b}$. Conditions: column, Buckyprep $(\varnothing 4.6 \mathrm{~mm} \times 250 \mathrm{~mm})$; eluent, toluene; flow rate, $1.0 \mathrm{~mL} / \mathrm{min}$; wavelength: $330 \mathrm{~nm} ; 40^{\circ} \mathrm{C}$.

The electron spin resonance (ESR) spectrum of $\mathbf{5 b}$ showed a unique octet signal (see Supporting Information File 1), indi- 
<smiles>COc1ccc(OC)c2c1COS(=O)C2</smiles>

1b toluene, $80^{\circ} \mathrm{C}$

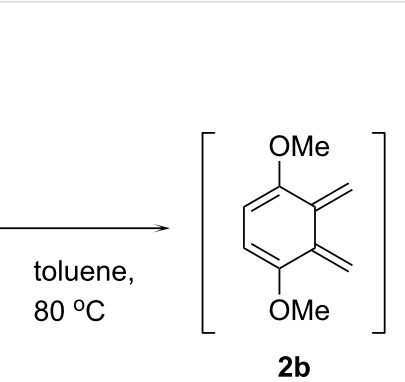

2b
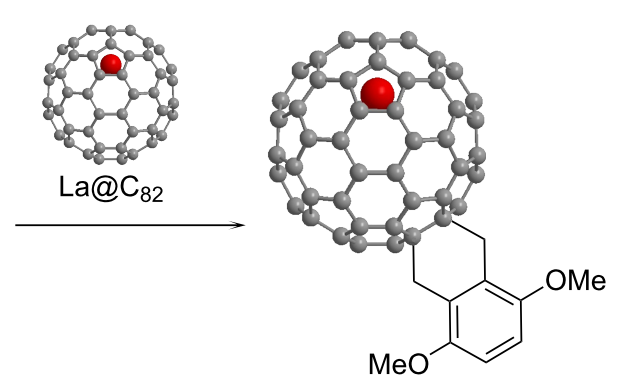

$5 b$

Scheme 2: Synthesis of $[4+2]$ adducts of $L a @ C_{82}$.

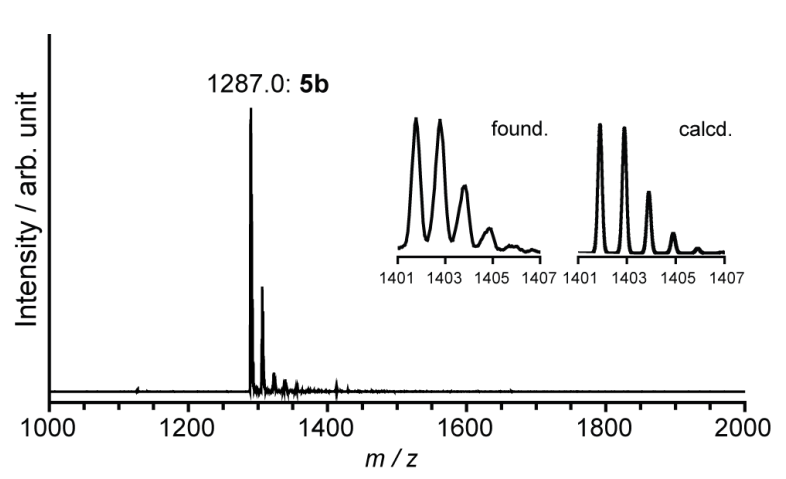

Figure 11: MALDI-TOF mass spectra (negative mode) of $\mathbf{5 b}$, using 1,1,4,4-tetraphenyl-1,3-butadiene as matrix.

cating the paramagnetic property of $\mathbf{5 b}$ as well as pristine La@ $\mathrm{C}_{82}$. This result also indicates that the cycloaddition of the $o$-quinodimethane does not lead to a remarkable change in the electronic properties of the fullerene. This fact is supported by the vis-NIR absorption spectrum of $\mathbf{5 b}$, which retains the specific absorption bands of the pristine La@ $\mathrm{C}_{82}$ (Figure 12). Broadening of the absorption bands is also observed, which is expected to be caused by the reduction of the molecular symmetry from $C_{2 v}$ to $C_{1}$ (vide infra).

Further characterization of the molecular structure was performed using NMR measurements. Because $\mathbf{5 b}$ has an openshell electronic structure as well as pristine $\mathrm{La} @ \mathrm{C}_{82}, \mathbf{5 b}$ was reduced electrochemically by one electron using bulk potential electrolysis for the NMR measurements. ${ }^{1} \mathrm{H}$ NMR spectrum of the resulting anionic $\mathbf{5 b}\left([\mathbf{5 b}]^{-}\right)$clearly illustrates the characteristic signals from the addend (Figure 13). Signals of the methylene protons appear as a sharp $\mathrm{AB}$ system at 4.55, 4.30, 2.94, and $2.82 \mathrm{ppm}$. The ${ }^{13} \mathrm{C}$ NMR spectrum demonstrates the total sum of 82 signals from the carbon cage (Figure 14), indicating $C_{1}$ molecular symmetry for $[\mathbf{5 b}]^{-}$. Signals at 58.2 and $56.0 \mathrm{ppm}$ are attributed to the $\mathrm{sp}^{3}$ carbons of the addition position of the addend.

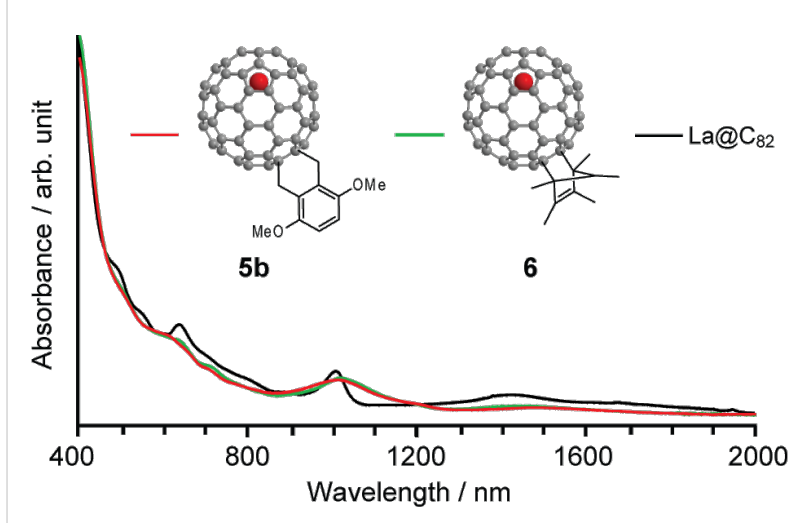

Figure 12: Vis-near-IR spectra of $5 b, 6$ and $L a @ C_{82}$ in $\mathrm{CS}_{2}$.

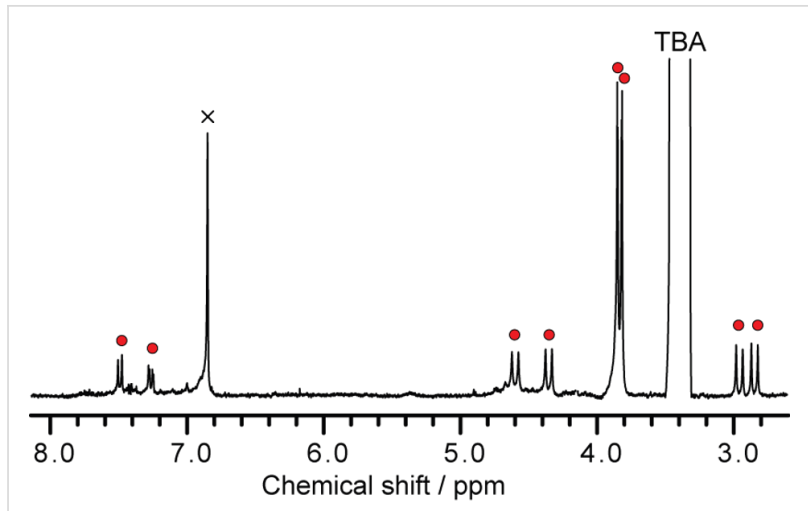

Figure 13: ${ }^{1} \mathrm{H}$ NMR spectrum of $[5 b]^{-}$in acetone- $d_{6} / \mathrm{CS}_{2}(3 / 1=\mathrm{v} / \mathrm{v})$ at $223 \mathrm{~K}$.

The vis-NIR absorption spectra provide information related to the molecular structure of $\mathbf{5 b}$ (Figure 12). The resemblance between the spectra of $\mathbf{5 b}$ and that of the previously reported [4 + 2] cycloadduct of La@ $\mathrm{C}_{82}\left(6: \mathrm{La} @ \mathrm{C}_{82} \mathrm{Cp}^{*}\right)$ [11] in the vis-NIR region imply the isostructural characteristics of the respective compounds, which show the same addition pattern of the substituents. Although the structure of $\mathbf{5 b}$ was not elucidated using X-ray crystallographic analysis, synthetic prece- 


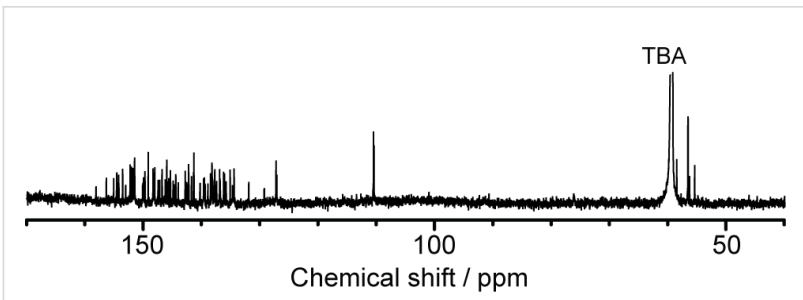

Figure 14: ${ }^{13} \mathrm{C}$ NMR spectrum of $[5 b]^{-}$in acetone- $d_{6} / \mathrm{CS}_{2}(3 / 1=\mathrm{v} / \mathrm{v})$

dents with absorption spectral data and theoretical calculations [16] strongly suggest that the most feasible addition site of the addend is that indicated in Scheme 2.

The thermodynamic stability of $\mathbf{5} \mathbf{b}$ was evaluated by thermal heating. When a toluene solution of $\mathbf{5 b}$ was let to stand at $30{ }^{\circ} \mathrm{C}$, no decomposition was observed, whereas 6 showed decomposition and generation of pristine La@ $\mathrm{C}_{82}$ (Figure 15). This result shows that using sultines, which generate reactive $o$-quinodimethanes and which afford cycloadducts, is an effective means to afford thermodynamically stable $[4+2]$ adducts of La@ $\mathrm{C}_{82}$.

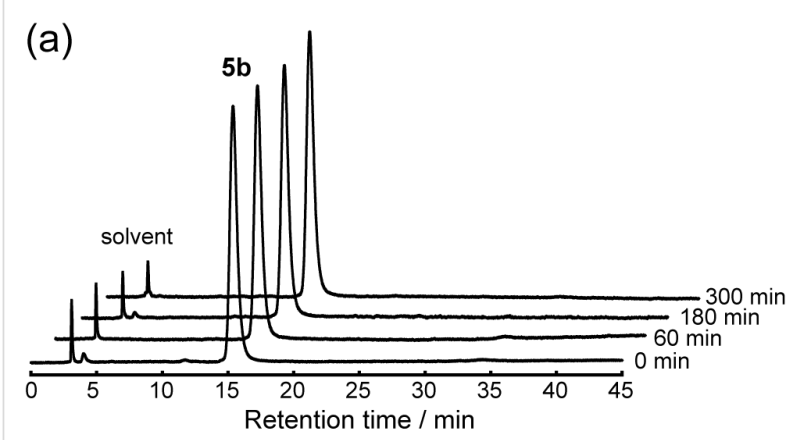

(a)

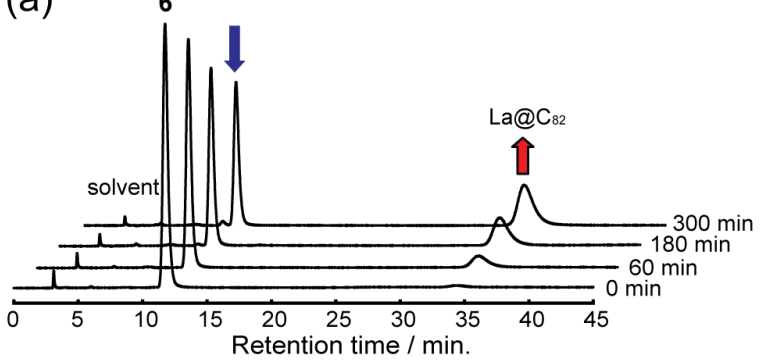

Figure 15: HPLC profiles for comparison of the thermal stabilities of (a) 5 b and (b) 6 at $30^{\circ} \mathrm{C}$. Conditions: column, Buckyprep $(\varnothing 4.6 \mathrm{~mm} \times$ $250 \mathrm{~mm}$ ); temperature, $40{ }^{\circ} \mathrm{C}$.

\section{Conclusion}

In summary, novel cycloadducts of $\mathrm{La}_{2} @ \mathrm{C}_{80}$ and $\mathrm{La} @ \mathrm{C}_{82}$ were synthesized efficiently by a $[4+2]$ cycloaddition reaction using sultines as a precursor of reactive $o$-quinodimethanes. Isolation of $\mathbf{3 b}$ and $4 \mathbf{a}$, respectively, was achieved by the selective thermal decomposition of unstable isomers. The thermal stability of $\mathbf{5 b}$ was also evaluated in comparison with $\mathbf{6}$, and $\mathbf{5 b}$ shows remarkable thermal stability. The molecular structures of the resulting compounds were characterized by spectroscopic analyses. Dynamic ${ }^{1} \mathrm{H}$ NMR spectroscopic investigations of $\mathbf{4 a}$ reveal temperature-dependent changes related to the conformational changes in the cyclohexane moiety generated upon reaction. The use of sultines for chemical modification of endohedral metallofullerenes has proved to be of general scope, being particularly useful to prepare thermally stable $[4+2]$ cycloadducts.

\section{Supporting Information}

Supporting information features detailed experimental procedures and spectral data for the compounds.

\section{Supporting Information File 1}

Descriptions on the synthesis and analyses of the compounds.

[http://www.beilstein-journals.org/bjoc/content/ supplementary/1860-5397-10-65-S1.pdf]

\section{Acknowledgements}

This work is supported by a Grant-in-Aid for Scientific Research on Innovative Areas (20108001, "pi-Space"), a Grantin-Aid for Scientific Research (A) (202455006), (B) (24350019) and Young Scientists (B) (25810098) from the Ministry of Education, Culture, Sports, Science, and Technology of Japan, The Strategic Japanese-Spanish Cooperative Program funded by JST and MINECO (Projects PLE-20090039 and PIB2010JP-00196), and by the European Research Council ERC-2012-ADG_20120216 (Chirallcarbon). The iCEMS is supported by the World Premier International Research Center Initiative (WPI), MEXT, Japan. N. M. thanks to Alexander van Humboldt Foundation.

\section{References}

1. Akasaka, T.; Wudl, F.; Nagase, S., Eds. Chemistry of Nanocarbons; Wiley: Chichester, 2010.

2. Chaur, M. N.; Melin, F.; Ortiz, A. L.; Echegoyen, L. Angew. Chem., Int. Ed. 2009, 48, 7514-7538. doi:10.1002/anie.200901746

3. Rudolf, M.; Wolfrum, S.; Guldi, D. M.; Feng, L.; Tsuchiya, T.; Akasaka, T.; Echegoyen, L. Chem.-Eur. J. 2012, 18, 5136-5148. doi:10.1002/chem.201102844

4. Popov, A. A.; Yang, S.; Dunsch, L. Chem. Rev. 2013, 113, 5989-6113. doi:10.1021/cr300297r

5. Nagase, S.; Kobayashi, K. J. Chem. Soc., Chem. Commun. 1994, 1837-1838. doi:10.1039/c39940001837

6. Kobayashi, K.; Nagase, S.; Akasaka, T. Chem. Phys. Lett. 1996, 261, 502-506. doi:10.1016/0009-2614(96)01026-3 
7. Hirsch, A.; Bettreich, M. Fullerenes, Chemistry and Reaction; Wiley-VCH: Weinheim, Germany, 2005.

8. Fu, W.; Zhang, J.; Fuhrer, T.; Champion, H.; Furukawa, K.; Kato, T.; Mahaney, J. E.; Burke, B. G.; Williams, K. A.; Walker, K.; Dixon, C.; Ge, J.; Shu, C.; Harich, K.; Dorn, H. C. J. Am. Chem. Soc. 2011, 133, 9741-9750. doi:10.1021/ja202011u

9. Lee, H. M.; Olmstead, M. M.; lezzi, E.; Duchamp, J. C.; Dorn, H. C.; Balch, A. L. J. Am. Chem. Soc. 2002, 124, 3494-3495. doi:10.1021/ja020065x

10. Maeda, Y.; Miyashita, J.; Hasagawa, T.; Wakahara, T.; Tsuchiya, T.; Nakahodo, T.; Akasaka, T.; Mizorogi, N.; Kobayashi, K.; Nagase, S.; Kato, T.; Ban, N.; Nakajima, H.; Watanabe, Y. J. Am. Chem. Soc. 2005, 127, 12190-12191. doi:10.1021/ja053983e

11. Maeda, Y.; Sato, S.; Inada, K.; Nikawa, H.; Yamada, M.; Mizorogi, N.; Hasegawa, T.; Tsuchiya, T.; Akasaka, T.; Kato, T.; Slanina, Z.; Nagase, S. Chem.-Eur. J. 2010, 16, 2193-2197. doi:10.1002/chem.200902512

12. Illescas, B. M.; Martín, N.; Seoane, C.; Ortí, E.; Viruela, P. M.; Viruela, R.; de la Hoz, A. J. Org. Chem. 1997, 62, 7585-7591. doi:10.1021/jo9706436

13. Segura, J. L.; Martín, N. Chem. Rev. 1999, 99, 3199-3246. doi:10.1021/cr990011e

14. Maroto, E. E.; de Cózar, A.; Filippone, S.; Martin-Domenech, A.; Suarez, M.; Cossío, F. P.; Martín, N. Angew. Chem., Int. Ed. 2011, 50, 6060-6064. doi:10.1002/anie.201101246

15. Yamada, M.; Wakahara, T.; Nakahodo, T.; Tsuchiya, T.; Maeda, Y.; Akasaka, T.; Yoza, K.; Horn, E.; Mizorogi, N.; Nagase, S. J. Am. Chem. Soc. 2006, 128, 1402-1403. doi:10.1021/ja056560l

16. Takano, Y.; Obuchi, S.; Mizorogi, N.; García, R.; Herranz, M. Á.; Rudolf, M.; Wolfrum, S.; Guldi, D. M.; Martín, N.; Nagase, S.; Akasaka, T. J. Am. Chem. Soc. 2012, 134, 16103-16106. doi:10.1021/ja3055386

\section{License and Terms}

This is an Open Access article under the terms of the Creative Commons Attribution License (http://creativecommons.org/licenses/by/2.0), which permits unrestricted use, distribution, and reproduction in any medium, provided the original work is properly cited.

The license is subject to the Beilstein Journal of Organic Chemistry terms and conditions:

(http://www.beilstein-journals.org/bjoc)

The definitive version of this article is the electronic one which can be found at: $\underline{\text { doi: } 10.3762 / \text { bjoc. } 10.65}$ 